\title{
Wie kam OSC in die Welt?
}

\section{Astrid Schreyögg}

Online publiziert: 19. Juli 2014

(C) Springer Fachmedien Wiesbaden 2014

\section{Der Vorlauf}

Die Supervision bildete den Ausgangspunkt für die Zeitschrift OSC. Sie war auch das erste der drei Formate, das ich im Studium als „Fallberatung“ im klinisch-psychologischen Kontext kennen lernte. Später etablierte ich an einer Fachakademie für Sozialpädagogik ein Supervisorensystem für Studierende, das zusammen mit einer Fachhochschule für Sozialarbeit bzw. deren Studierenden durchgeführt werden konnte. Unsere Studierenden wurden von den Studenten der FH für ihre Praktika supervidiert, und diese FH-Studenten erhielten dafür Lehrsupervision. Ich fand das Format fachlich sehr anregend und nahm Mitte der 1970er Jahre bei einem KonopkaSchüler an sozialarbeiterisch orientierter Gruppensupervision teil.

Später lernte ich Supervision in Therapieausbildungen kennen. Diese Supervision knüpfte zwar wieder an das an, was ich schon im Studium erfahren hatte, ich wurde aber zunehmend unzufrieden mit dieser klinischen Variante. Ich habe nämlich mehr als ein Jahrzehnt mit großem Vergnügen in organisatorischen Systemen gearbeitet und eines davon über große Strecken sogar geleitet. Aus dieser Erfahrung erschien mir die Art der Supervision, wie sie in therapeutischen Milieus üblich war, ziemlich verengt. Es ging dabei meistens um individuelle Phänomene von Klienten, deren Wirkung auf den Therapeuten und umgekehrt. Der Kontext, vor allem der organisatorische Kontext, blieb immer ausgespart, denn es mussten ja möglichst die Emotionen behandelt werden. Institutionalisierungen jeder Art erschienen nur als Behinderung. Deshalb nahm ich mir vor, Supervision möglichst aus ihrer ,therapeutischen Umklammerung“ zu befreien.

\footnotetext{
A. Schreyögg $(\bowtie)$

Berlin, Deutschland

E-Mail: info@schreyoegg.de
} 
Seit Mitte der 1980er Jahre war ich als freiberufliche Psychotherapeutin und Lehrtherapeutin tätig, die Supervision fesselte mich aber letztlich mehr. Seit 1982 hatte ich begonnen, Ausbildungsgruppen für Supervision in Österreich, danach in Deutschland, Südtirol und der Schweiz für die EAP (Europäische Akademie für Psychosoziale Gesundheit/Fritz Perls Institut) zu leiten.

Hier möchte ich eine Anekdote einflechten: Im Jahr 1989 wurde von etlichen Ausbildungsinstituten für Supervision verlautbart, dass Sie einen Verband für Supervision gründen wollen (die DGSv). Das fand ich prima und verließ deshalb eigens eine Konferenz in Wien, um an der Gründung in Köln teilzunehmen. An dem entsprechenden Ort fand ich eine große Runde vor mit Wolfgang Weigand, Heinz Kersting und anderen Größen der deutschen Supervisionsszene. Mehrere dieser Herren teilten mir sofort empört mit, dass ich nicht eingeladen sei und überhaupt als Vertreterin eines Therapie-Instituts hier nichts zu suchen habe. Enttäuscht verließ ich die Versammlung und nahm mir vor, einen eigenen Verband zu initiieren. Etliche Jahre später ist es mir tatsächlich gelungen, den „Supervisor BDP“ beim Berufsverband Deutscher Psychologinnen und Psychologen als Gegenstück zum „Supervisor DGSv“ zu etablieren. Heute gibt es aber freundliche Kooperationen zwischen beiden Verbänden, und ich wurde sogar als ,verdientes Mitglied“ von der DGSv aufgenommen.

Für die Leitung meiner Ausbildungsgruppen schleppte ich jeweils große Mengen an Skripten und Büchern mit, um die Ausbildungskandidaten fachlich gut zu versorgen. Als ich mich wieder einmal so abschleppte, kam mir die Idee, dass ich ein Lehrbuch für die angehenden Supervisoren schreiben sollte. Diese Idee rumorte noch eine Weile in mir, bis ich mich entschied, dieses Vorhaben als Dissertation zu realisieren. Zum Glück fand ich schnell einen Doktorvater, der allerdings mit der Bemerkung: „Ein Lehrbuch kann ja jeder schreiben“, noch eine empirische Evaluation meiner Arbeit verlangte. 1991 war das Werk vollendet. Der Junfermann-Verlag in Paderborn, den ich zuerst angesprochen hatte, wollte zunächst nur das Lehrbuch, die Evaluation erschien dann gesondert zusammen mit einem Didaktik-Teil.

Der Vorteil dieser „Fleißarbeit“ lag auf verschiedenen Ebenen: Zum einen hatte ich einen hübschen Titel erworben, zum anderen hatte ich mir einen umfassenden Eindruck verschafft, was Supervision ist, wer in diesem Feld was denkt und wie man es noch weiter ausbauen könnte. Ein weiterer Vorteil war, dass ich mit diesem Lehrbuch einen konzeptionellen Markstein gesetzt hatte. Dieses Buch ist 2010 in der 5. Auflage im VS Verlag erschienen und erfreut sich weiterhin einer breiten Leserschaft. Und schließlich war ich durch die intensive Beschäftigung mit dem Thema „Supervision“ auch in der Lage, sehr hochwertige Ausbildungsprogramme zu diesem Format zu kreieren. Diese führte ich dann zusammen mit etlichen Kolleginnen und Kollegen zuerst bei der Europäischen Akademie für psychosoziale Gesundheit durch, seit Mitte der 1990er Jahre bei der Deutschen Psychologen Akademie und im Weiteren für Psychologen der Bundesanstalt für Arbeit sowie für Bayerische Schulpsychologen.

\section{Die eigentliche Geburt von OSC}

Als ich wieder eines dieser Ausbildungsprogramme leitete, das sich ausschließlich aus Gestaltpsychotherapeuten rekrutierte, meinte ich so leichthin, dass man eigent- 
lich eine gute Fachzeitschrift für Supervision ins Leben rufen müsste, denn das bisherige, einzige Blatt zur Supervision sei doch etwas einseitig. Unversehens stieß ich bei den Teilnehmerinnen und Teilnehmern auf große Resonanz. Besonders ein Gruppenmitglied, Christoph Schmidt-Lellek, der schon etliche Jahre bei Junfermann als professioneller Lektor gearbeitet hatte (u. a. als Redakteur der Zeitschrift „Integrative Therapie“), meinte sofort, dass er gerne als Redakteur und Mitherausgeber fungieren wolle. Auch drei andere Kollegen, mit denen wir kooperierten, fanden die Idee gut. Erste Verhandlungen mit Junfermann führten allerdings nicht zum Erfolg. Mein damaliger Ko-Trainer aber, Wolfgang Rechtien von der FernUni Hagen, der gerade die Redaktion der Gruppendynamik für den Verlag Leske \& Budrich managte, hatte den Tipp, dass in diesem Verlag vielleicht auch eine Zeitschrift mit dem Schwerpunkt Supervision erscheinen könne. Wir hatten uns vorher überlegt, dass das Format Supervision für eine solche Zeitschrift vielleicht zu schmal sei. Ich warf ein, dass man auch die Organisationsberatung berücksichtigen solle, während Hilarion Petzold noch die Idee hatte, „Clinical Management“ als dritten Begriff zu nehmen. Diese Kombination erschien uns sehr sinnvoll.

So fuhren wir - Wolfgang Rechtien, der den Kontakt angebahnt hatte, Christoph Schmidt-Lellek, Hilarion Petzold, Leiter der EAP, Nando Belardi und ich - im Frühjahr 1994 zu fünft zu Herrn Budrich nach Opladen. Er war ein eindrucksvoller alter Herr mit einem großen Hund zwischen riesigen Bücherwänden. Ich legte ihm das Konzept vor, er überflog es (denn ich hatte ihm schon vorher ein Exemplar übersandt). Er meinte: „Warum nicht, aber die muss schon viermal im Jahr erscheinen. Bei zweimal vergessen die Leute die Zeitschrift schnell. Außerdem muss die eine Abkürzung haben, sonst ist der Titel zu sperrig.“ „Ja“, meinte ich, „wir könnten sie OSC nennen.“ „Na ja“, meinte er grinsend, „das hört sich zwar irgendwie nach ,Oberbergischem Sportclub“ an, aber warum nicht.“ „Wer von Ihnen wird der Herausgeber sein? Fünf geht ja nicht, das muss einer sein.“ „Ich will das machen, ich hatte die Idee“, sagte ich, ,die anderen fungieren aber als Mitherausgeber. Außerdem wollen wir einen wissenschaftlichen Beirat installieren.“ „Ja gut, so kann das gehen“, meinte er. Und so legten wir los. Wir trafen uns noch ein einziges Mal zu fünft für die Planung der kommenden Hefte.

\section{Warum OSC ihren Namen und den Verlag wechselte}

Gegen Ende der 1990er Jahre ergaben sich in der Beratungsszene neue Entwicklungen. Zur Supervision schien irgendwie alles gesagt, in der Organisationsberatung leuchtete der Stern OE auch nicht mehr so hell wie früher. Und zum Thema „Clinical Management“ bekamen wir kaum Aufsätze. Dafür tauchte Coaching als neues, aussichtsreiches Format auf. Zwar hatten wir schon in der ersten Ausgabe von OSC einen Beitrag von Maren Fischer-Epe über ein Coaching-Beispiel, den eigentlichen Schub erhielt dieses Format aber erst gegen Mitte bis Ende der 1990er Jahre. Aus der Erfahrung, dass sich die Fragestellungen vieler meiner Teamsupervisionen in Drogeneinrichtungen, Fachkliniken usw. um die jeweilige Führungskraft zentrierten, hatte ich schon 1995 bei Campus ein Lehrbuch für Coaching herausgebracht und zeitgleich eine erste Ausbildungsgruppe für Coaching durchgeführt. 
Zum Thema Coaching gab es jetzt auch entsprechende Kongresse. Auf einem dieser Kongresse traf ich Thomas Webers, damals Redakteur der ,Wirtschaftspsychologie“. Ich sagte: „Eigentlich müsste es eine Zeitschrift für Coaching geben.“ Darauf meinte er: „Euer C in OSC kann doch auch für Coaching stehen.“ „Mensch, das ist eine prima Idee.“ Flugs rief ich alle Mitherausgeber an, ob sie das auch finden. Ja, das fanden sie auch. Und so steht seit Heft $1 / 2001$, also dem 8. Jahrgang, das C in OSC für Coaching. In den nachfolgenden Jahren bildete Coaching tatsächlich zunehmend einen Schwerpunkt von OSC.

Etwas später erfuhren wir zunächst gerüchteweise, dass Herr Budrich seinen Verlag verkaufen wollte. Als ich ihn am Telefon spaßeshalber fragte, ob er wohl insolvent sei, meinte er, ,nein, es ist umgekehrt, wenn ein Verlag am besten floriert, soll man ihn verkaufen.“ „Du meine Güte“, dachte ich, ,jetzt wird es brenzlig“, denn ich wusste, dass Verlage mit Zeitschriften meistens kein großes Geschäft machen können. Aber nein, es entwickelte sich ganz anders: Herr Budrich hatte seinen Verlag an den VS Verlag für Sozialwissenschaften verkauft. So erscheint die OSC seit dem 11. Jahrgang 2004 im VS Verlag, der seinerseits seit $2010 \mathrm{zu}$ dem Imperium der wissenschaftlichen Springer-Verlage gehört und deshalb seit 2012 als „Springer VS“ firmiert.

\section{Personelle Verschiebungen im Mitherausgeberstab und im wissenschaftlichen Beirat}

Im Verlauf der Entwicklung von OSC ergaben sich auch personelle Veränderungen. Bis zum Jahr 2010 rekrutierte sich der Mitarbeiterstab noch aus den OSC-Gründern. $\mathrm{Ab}$ 1/2010 schieden aber Nando Belardi und Wolfgang Rechtien aus dem Mitherausgeberstab aus und wechselten in den wissenschaftlichen Beirat. Dafür gewannen wir Heidi Möller, Beate Fietze und Ferdinand Buer als neue Mitherausgeber. Ferdi blieb uns allerdings leider nur wenige Jahre erhalten, obschon er auch immer sehr profunde Beiträge geliefert hat. Er verabschiedete sich schon nach drei Jahren, also im Jahr 2013. Wir konnten dann allerdings im gleichen Jahr Siegfried Greif als Mitherausgeber begrüßen. Im Übrigen gewinnen wir immer mal wieder Gastherausgeber für einzelne Ausgaben von OSC, wie z. B. Arist v. Schlippe und Tom Rüsen über die „Beratung von Familienunternehmen in Wendepunkten“ (4/2007) oder Cornelia Seewald über „Coaching im Mittelstand“ (3/2013).

Unser wissenschaftlicher Beirat setzt sich nach wie vor aus Kolleginnen und Kollegen aus Deutschland, Österreich, der Schweiz, den Niederlanden und sogar aus Spanien zusammen. Bei der Zusammensetzung haben Christoph und ich immer darauf geachtet, dass er nicht nur regionale Pluralität widerspiegelt, sondern auch in fachlicher Hinsicht eine Mischung ist. 


\section{OSC als Forum für die OSC-Themen}

Durch den Verkauf von Leske \& Budrich erschienen natürlich zunächst alle Publikationen aus dem Bereich der Supervision in den weiteren Auflagen im VS Verlag. Wenn aber Bücher mit bestimmten Themen bei einem Verlag erscheinen, zieht das auch ähnliche Themen an. So platzieren heute immer mehr Autoren aus dem Themenbereich Supervision und Coaching ihre Publikationen bei VS bzw. nun Springer-VS. Mir wurde sogar die Möglichkeit eröffnet, eine Reihe für Supervision und Coaching ins Leben zu rufen, die ich 2008 mit einem Buch über „Coaching für die neu ernannte Führungskraft" eröffnet habe. OSC fungiert aber für diese Themen auch als Forum. Der Verlag nutzt die Zeitschrift jeweils als Werbeträger, um in den Coverseiten über einschlägige Neuerscheinungen zu informieren.

Wie Reinald Klockenbusch in seinem Beitrag anmerkt, hat sich OSC unter verlegerischen Gesichtspunkten ausgesprochen gut entwickelt. Das heißt, es erbringt auch einen ordentlichen Ertrag für den Verlag. Für uns Herausgeber hat OSC aber auch unter fachlichen Gesichtspunkten einen hohen Stellenwert, gelingt es uns doch, über diese Zeitschrift auch Innovationen in den Bereichen Coaching und Supervision anzustoßen. So konnten wir z. B. immer wieder Beiträge gewinnen über Beratung in unterschiedlichen Behörden. Auch das interne Coaching war ein Thema, das bislang allzu selten in Erscheinung trat. Daneben haben wir natürlich immer wieder Supervision und Coaching in neuen Feldern aufgegriffen, so etwa Coaching in der Hochschule, in Familienunternehmen, in der Schule, in der Altenarbeit, in Kliniken usw. Es gab auch zielgruppenspezifische Themen wie „Coaching von Frauen“ oder „die Beratung von Männern im Beruf", des Weiteren interkulturelle Themen oder "Gesundheit im Beruf". Gelegentlich gab es sogar fachliche Diskurse, die über mehrere Ausgaben von OSC geführt wurden, wie etwa der über Teamsupervision zwischen Wolfgang Schmidbauer und mir im Jahr 2003 oder der über Paradoxien der Wertschätzung zwischen Mirko Zwack et al. auf der einen Seite und Ferdinand Buer auf der anderen in den Jahren 2011 und 2012. Solche fachlichen Kontroversen würden wir auch in den kommenden Jahren gerne wieder bringen.

Wichtig waren uns neben konzeptionellen und empirischen Arbeiten sowie qualifizierten Praxisberichten auch immer wieder Auseinandersetzungen über ethische und normative Fragestellungen. Für manche dieser Themenschwerpunkte haben wir als neues Forum die „Sonderhefte von OSC“ genutzt. Das erste hieß „Konzepte des Coaching“ (2007), das zweite „Praxeologie des Coaching“ (2008), das dritte befasste sich mit „Organisation im Coaching“ (2009), und das vierte war „Philosophie, Ethik und Ideologie in Coaching und Supervision“" (2011) gewidmet. Die Produktion dieser Hefte haben wir in Absprache mit dem Verlag allerdings nicht fortgeführt. Zum einen war das ein zusätzlicher, großer Arbeitsaufwand für uns, der zum anderen bei manchen Lesern Ärger hervorrief, denn als Abonnenten von OSC ging ihnen ja nun ein ganzes Buch zu, das sie nicht bestellt hatten, aber nun zurücksenden oder bezahlen mussten. 
So haben wir uns für die nächsten Jahre vorgenommen, auch weiterhin innovative Themen aufzuspüren, diese aber in Form von den üblichen vier Heften pro Jahr unterzubringen. Und das wollen wir auch weiterhin mit viel Schwung und Innovationslust machen. 